\title{
Labyrinthe
}

17 | 2004 (1)

Jacques Rancière, l'indiscipliné

\section{Rancière sociologue, autrement}

\section{Déborah Cohen}

\section{OpenEdition}

Journals

Édition électronique

URL : http://journals.openedition.org/labyrinthe/180

DOI : 10.4000/labyrinthe.180

ISSN : 1950-6031

Éditeur

Hermann

Édition imprimée

Date de publication : 15 avril 2004

Pagination : 97-101

Référence électronique

Déborah Cohen, «Rancière sociologue, autrement », Labyrinthe [En ligne], 17 | 2004 (1), mis en ligne le 13 juin 2008, consulté le 19 avril 2019. URL : http://journals.openedition.org/labyrinthe/180 ; DOI :

$10.4000 /$ labyrinthe. 180

Propriété intellectuelle 


\section{RANCIÈRE SOCIOLOGUE, AUTREMENT}

Déborah CoHEN

Si Jacques Rancière s'est plu à jouer entre les disciplines, refusant d'endosser la figure du philosophe, de l'écrivain ou de l'historien professionnels, n'assumant aucune exclusive mais ne rejetant aucun parcours, pour se situer toujours dans l'entre-deux, il est, en apparence au moins, deux disciplines qu'il ne revendique jamais, c'est la sociologie et l'anthropologie. La première est avant tout interrogée à partir de l'œuvre de Pierre Bourdieu, avec laquelle Rancière marque d'autant plus nettement les distances qu'il en partage bien des thèmes; la seconde est plutôt considérée à travers l'usage qu'ont pu en faire les historiens recourant à la notion de «mentalités ». On peut résumer les reproches qu'il adresse à ces deux disciplines en les regroupant sous l'idée de propriété. L'analyse en termes de propriétés a deux principaux défauts au yeux de Rancière. D'une part, une propriété est une qualité qui se maintient dans la durée et tend donc asymptotiquement à échapper aux changements de l'histoire et à se transformer en nature. Elle devient ainsi cause, élément autonome d'explication. À l'origine de la reproduction sociale on peut alors placer les groupes dominés eux-mêmes et leurs caractéristiques acquises: c'est ce que font les tenants de l'interprétation culturelle de la pauvreté. Pour Oscar Lewis par exemple, la pauvreté engendre une culture spécifique, système rationnel d'autodéfense né de l'adaptation à un ensemble de conditions extérieures, qui est incorporé et se perpétue de génération en génération $^{1}$. Si Bourdieu ajoute à cette analyse en démontant les mécanismes par lesquels cette incorporation se pare d'une illusion de liberté qui la rend possible, c'est d'abord au scandale d'une inéluctabilité, d'une loi de nature également présente chez Bourdieu, que s'attache Rancière. Que faire, nous dit-il, « d'une science des rapports de force qui les dit

1. « La vida », une famille portoricaine dans une culture de pauvreté, San Juan et New York, traduit par Jean Rosenthal, Paris, Gallimard, 1969. 
infrangibles? » $(P P, 259)$. D'autre part, les propriétés sont toujours analysées en grappes: le sujet en possède plusieurs, toutes reliées les unes aux autres et formant un ensemble cohérent, une totalité à la signification unique. Rien donc pour déranger le bel ordonnancement, pas de faille mais un univers où chaque partie renvoie au tout, un système clos, « comme si la science n'assurait sa différence qu'à postuler la solide identité à soi » de chaque sujet $(N P, 10)$.

Un certain nombre de sociologues critiques de Bourdieu se sont attaqués précisément à l'idée de cohérence interne des qualités formant l'habitus et à celle de continuité de la personne dans le temps. C'est notamment le cas de Bernard Lahire qui a montré la pluralité des rôles sociaux assumés par un même individu sur différentes scènes et la facilité avec laquelle on recourait à l'un ou l'autre selon leur adéquation contextuelle, en dépit même de leur caractère éventuellement contradictoire, inhibant les dispositions non pertinentes ${ }^{2}$. Voilà l'individu échappant en effet au monolithisme d'une formule, d'un destin. Cela suffirait-il à répondre aux inquiétudes de Rancière face aux propos de la sociologie critique? Tout n'est pourtant pas si simple:

Moi, qui ai passé pas mal de temps à critiquer Bourdieu [déclarait Rancière sans faire allusion à aucun discours en particulier], quand je vois la manière dont certains aujourd'hui le traitent comme un penseur dépassé, j'ai envie de dire que c'est quand même moins dépassé que ce qu'on veut mettre à la place ${ }^{3}$.

La sociologie psychologique de Lahire a tout de même ceci de problématique (outre le fait qu'elle ne s'est appuyée empiriquement que sur des cas « appartenant essentiellement aux classes moyennes et supérieures » propres à exemplifier au mieux la traversée permanente de contextes sociaux différenciés ${ }^{4}$ ) qu'elle fait fi de l'ampleur des contraintes imposées de manière continue quel que soit le contexte. À

2. L'Homme pluriel. Les ressorts de l'action, coll. « Essais et Recherches », Paris, Nathan, 1998. Voir aussi son article « De la théorie de l'habitus à une sociologie psychologique », dans Bernard Lahire (dir.), Le Travail sociologique de Pierre Bourdieu. Dettes et critiques, Paris, La Découverte, 2001.

3. «La question sociale: visions de sociologues, visions d'historiens. Table ronde », Vie sociale $\mathrm{n}^{\circ} 6$, novembre-décembre 1996, p. 471.

4. Bernard Lahire, Portraits sociologiques. Dispositions et variations individuelles, coll. « Essais et Recherches », Paris, Nathan, 2002. 


\section{Rancière sociologue}

force de vouloir éliminer l'idée de mentalité et de contexte englobant, elle présente un monde d'où sont absentes les violences symboliques de cette fondamentale "répartition des parts et des places » que Rancière désigne comme «partage du sensible» $(P S, 12)$. Ce qui s'absente alors, c'est le tort, la souffrance, celle de l'ouvrier poète que, précisément, on ne peut voir comme poète, parce qu'il n'y a pas de scène sociale, de contexte pour ce faire. Alors, certes, la classe ne peut être pensée comme un ensemble de propriétés, mais on ne saurait se contenter de cette « fable du rapport des individus au tout, faite pour liquider le rapport litigieux des parties » (Més., 115).

Prendre en compte le fondamental partage du sensible, c'est refuser une sociologie qui résout le problème posé par la tendance naturalisante et totalisante de l'habitus en multipliant les lieux et valeurs à disposition d'un acteur libre d'en jouer à sa guise. C'est refuser l'illusion de justice et de paix que comporterait la réduction du litige à des situations de disputes marquées par « des efforts de convergence au cœur même du différend ${ }^{5} »$. Car si ces efforts peuvent prendre appui sur une pluralité d'univers de valeurs partagées (ce que Boltanski et Thévenot appellent des « cités »), il n'en reste pas moins que les parties impliquées sont pré-déterminées, y compris dans ce que ces auteurs désignent comme leur « taille», c'est-à-dire leur place telle qu'elle est définie en fonction du système de la cité mobilisée ${ }^{6}$. Rancière nous rappelle qu'il est, au-delà de propriétés internes, un autre instrument pour penser les identités, celui du jugement. S'il y a quand même des classes sociales chez Rancière, c'est parce que s'impose la violence d'un ordre qu'il nomme «police »,

ordre des corps qui définit les partages entre les modes du faire, les modes d'être et les modes du dire, qui fait que tels corps sont assignés par leur nom à telle place et à telle tâche; c'est un ordre du visible et du dicible qui fait que telle activité est visible et telle autre ne l'est pas, que telle parole est entendue comme du discours et telle autre comme du bruit (Més., 52).

5. Luc Boltanski et Laurent Thévenot, De la justification, Paris, Gallimard, 1991, p. 26.

6. Sur l'intérêt de Rancière pour les acteurs multidimensionnels mais aussi sa critique de l'illusion d'une sortie de l'habitus qui n'est que mobilité individuelle entre des assignations identitaires non remises en question, voir « Entretien avec Jacques Rancière. Les hommes comme animaux littéraires », Mouvements, n 3, mars-avril 1999, p. 133-144. 
Cependant, nous n'avons pas à nous enfermer dans l'alternative des propriétés ou du jugement. Rancière prend acte des effets réels des identités assignées, mais nous convie aussi vers ce qui est exclu de la sociologie: «Une détermination qui ne serait ni dedans ni dehors, ni une propriété de la chose, ni un caractère du jugement sur la chose » $(A B, 130)$.

Ainsi, qu'il y ait des constructions sociales cohérentes et des identités contraignantes, Rancière le maintient envers et contre tous les reflux actuels. Pour autant, il ne nous laisse pas croire que c'est en comptant les parties du monde social que l'on pourra répondre à ce qui est le souci de la sociologie : «Qu'est-ce qui fait lien social"? ? Ce lien, qu'il définit comme l'objet de recherche de la sociologie, c'est aussi d'une certaine manière, sous le nom de politique, le souci propre de Jacques Rancière, mais avec la conscience qu'on ne le trouvera pas en creusant les identités sociales, communautaires; ce serait simplement « reconduire » la violence du partage établi du sensible, qui ne laisse pas de place à une rencontre (litigieuse) entre les classes. Il faut un mouvement de sortie de cette logique policière; mouvement que Rancière nomme de «subjectivation » et que l'on peut décomposer en deux temps, en réalité indissociables et noués: une déliaison tout d'abord, ou « désidentification » par laquelle on échappe à la définition policière des parties de la société - ne plus être l'ouvrier réduit à un corps-outil, une inhumanité, ne plus être le Français derrière son gouvernement colonialiste; et une «identification impossible » à l'autre qui est pourtant l'égal - impossible en partie précisément parce que ce que décrit la sociologie existe et détermine des identités. À ce sujet qui s'est placé dans l'entre-deux $(A B, 88)$, dans l'atopie de Socrate, des ouvriers poètes ou d'Ingrid Bergman dans Europe 51 - internée faute de n'être désormais ni de la bourgeoisie ni de la chaîne $(C V)$-, il faut un nom. Par exemple: prolétaire, républicain, révolutionnaire, damné de la terre, femme; mais ce nom ne peut renvoyer qu'à une abstraction; il ne désigne pas une partie du monde social, seulement la qualité de celui qui se tient sur ce lieu de l'entre-deux, du conflit, aussi longtemps qu'il s'y tient en faisant «comme si » il s'agissait réellement d'un monde commun reconnu.

7. « La question sociale...», art. cit., p. 446. 


\section{Rancière sociologue}

Si le sujet social existe, le sujet politique, lui, n'a pas de corps, pas d'autre lieu que le non-lieu du discours. Que devient alors chez Rancière la figure, traditionnellement associée au sujet politique par nos modernes républiques, celle de la représentation ? Claude Dubar a analysé en termes de crise les formes de représentation sur une scène que Rancière nommerait policière: en l'absence de définition communautaire de soi, le sujet ne se reconnaît plus en des chefs «naturels », il lui faut un représentant du tout qui soit, ce qui est problématique, en accord avec la représentation mobile qu'il a de lui-même. À partir du moment où la politique n'est plus une question de « communauté » ou de «statut », se demande Dubar, comment éviter ce qui paraît être la seule alternative à sa disparition même (ou en participe déjà), celle qui fait de la politique une question personnelle ${ }^{8}$ ? En rappelant que le sujet politique existe comme une fiction, Rancière indique un chemin qui tourne le dos non seulement à la course présente qui tente de faire coïncider l'image du représentant et l'image que le sujet individuel a de lui-même, mais aussi à l'idée de trouver un porte-parole des masses, tout particulièrement proche du mode de vie de ses « supposés semblables ». Sa politique n'est pas pour autant l'exigence (que d'aucuns diraient irréaliste) d'une mobilisation directe et permanente de tous et de chacun. Elle est refus d'une représentation qui ne serait que recherche d'une adéquation entre deux images (celle d'un moi ou d'un collectif et d'une instance tierce mais qui serait leur semblable); elle est élection de ceux qui ne sont pas une image mais une parole par laquelle ils « ont quelque chose à représenter aux bourgeois », aux autres groupes institués par la police $(N P, 9)$. Le litige et la politique sont possibles non pas là où paraît une parole propre mais où circule et fait lien une parole hybride qui fait « comme si » elle était commune, mettant en avant, sur fond de toutes les différences individuelles et collectives (en particulier de pouvoir et de ressources) le postulat fondamental d'une égalité comme êtres parlants.

8. Voir La Crise des identités. L'interprétation d'une mutation, Paris, Puf, 2000, chapitre 4, notamment p. 156-160. 